\title{
The prognostic role of circulating tumor cells in heavily pretreated individuals with a low life expectancy
}

\author{
Justin Stebbing ${ }^{*, 1}$, Victoria Harding ${ }^{1}$, Catherine EUrch', Thomas Kaier', \\ Guy Schofield', Matthew Flook', Constantine Alifrangis' ${ }^{1}$, Anna-Mary \\ Young ${ }^{1}$, Jacqueline A Shaw ${ }^{2}$, R Charles Coombes ${ }^{1}$ \& Jonathan Krell ${ }^{1}$
}

\begin{abstract}
Aims: Studies of circulating tumor cells (CTCs) have generally recruited individuals with newly diagnosed metastatic cancer, with recent data also indicating their prognostic value in the adjuvant setting. Their role in dying patients has not been established. Experimental: CTCs were measured in 43 individuals with metastatic breast cancer estimated to have less than 6 months to live who had exhausted standard therapeutic options. Results: Those with a CTC count of $\leq 100$ had a median of 182 days to live, compared with those with a CTC count of $>100$ who had a median of 17 days until death $(p=0.009$, Log rank, HR: 3.1, 95\% Cl: 1.4-7.3). Conclusion: A CTC count of $>100$ is associated with imminent death. Provided external validity is demonstrated, such information would be useful for patients and their families who often request specific prognostic clarity and could improve the quality of end-of-life care.
\end{abstract}

The majority of individuals with metastatic cancer request information regarding their survival duration, especially when their deaths are approaching [1,2]. Ideally, such information should be as realistic as possible; evidence indicates that patients prefer an honest and individualised approach in this context including detailed and specific information when discussing prognosis [3]. However, knowing these data and underlying principles is quite different to their application in every day clinical practice: when confronted by standard versions of the simple question "how long have I got?" we struggle to find suitable answers.

One of the most relevant reasons that we have difficulty in communicating information regarding life expectancy is a lack of obvious data on which to base our estimates. Clinical trials, typically our best resource for such data, generally recruit fitter patients undergoing their first-, second- or third-line therapy for cancer. Individuals with a poor performance status are almost always excluded, as are those with less than 3 months to live. Even with this information, communicating median survival times to patients is commonly misinterpreted. Few patients have sufficient understanding of statistics to appreciate the term median, for example, and fewer still understand interquartile range or $95 \%$ confidence intervals [4-7].

The use of circulating tumor cells (CTCs) in breast and other cancers has gained traction to provide prognostic information, especially with standardization of their measurement [8]. Most of the data for use of CTCs derives from studies in first line metastatic disease [9-12], and recent evidence has also indicated their utility in the adjuvant setting [13-16]. The CTC cut offs of $>5$ CTCs and $>2$ CTCs per $7.5 \mathrm{ml}$ of blood have been used to delineate a statistically significant worse overall survival in both settings, respectively. There are few data on their use at the end of life, when families

\section{KEYWORDS}

- biomarker • cancer

- circulating tumor cell $\bullet$ cut-off $>100 \bullet$ life expectancy $\bullet$ hospice - overall survival • palliative

'The Departments of Medical Oncology \& Palliative Care, Imperial College \& Imperial College Healthcare NHS Trust, Fulham Palace Road, London, W6 8RF, UK

2Department of Cancer Studies \& Molecular Medicine, University of Leicester, Leicester Royal Infirmary, Leicester, UK *Author for correspondence: Tel.: +44 203311 8295; Fax: +44 203311 1433; j.stebbing@imperial.ac.uk

\section{Future Medicine ${ }_{\text {part of }}$}


often ask for a specific prognosis in days, weeks or months, yet we are usually unable to provide objective information. Such information would however help patients and their families make appropriate plans, such as deciding between hospital, hospice or home as a place to be, or on the other hand whether the prognosis may be better than expected and one could even consider further therapy, a Phase I or other drug trial.

We therefore investigated use of CTCs to provide specific prognostic information, in individuals considered to be dying from metastatic breast cancer.

\section{Methodology}

Between May 2011 and February 2012, consecutive individuals with metastatic breast cancer admitted to the oncology unit at Imperial College Healthcare NHS Trust were considered eligible for CTC measurement provided two senior physicians considered they had less than 6 months to live. Patients included had an ECOG performance status of 2, 3 or 4 and had exhausted conventional lines of therapy. Appropriate locoregional ethics committee approval was obtained.

CTCs were measured using a single $7.5 \mathrm{ml}$ blood test with the Janssen Diagnostics CellSearch system as we have described [17-19]. Unlike our research studies, after the CellSave sample was taken it was left in a specific protected package for a courier to collect it that day or the next day and transport it to a designated CTC testing centre in Nottingham ( 120 miles from the London site).

All outcomes data were presented descriptively as means, medians or proportions. Given the observational nature of the study, no formal sample size or power analysis was undertaken; a total of 50 tests were provided free of charge by Janssen Diagnostics. Therefore, the most relevant outcomes from the statistical analysis are the $95 \%$ confidence intervals (CI). Survival curves from the day the CTCs were measured until death were generated by the method of Kaplan-Meier and compared between groups (i.e., CTCs $>100$ vs $\leq 100$ by the Log-rank test). The cut-off of 100 CTCs was chosen and prespecified as the only cut-off herein based on previous data suggesting that $100 \mathrm{CTCs}$ represents an 'oncologic emergency' [20]. In a supporting exploratory analysis, the relative risk of death between groups was estimated using Cox Proportional Hazard regression. Before the full regression analysis was initiated, the relevant covariates for initial model inclusion were identified by a univariate screening process with a preset alpha $=0.25$. The intent of this approach is to remove weak prognostic covariates so that a more manageable set of variables can be submitted to multivariate techniques. Independent prognostic variables with a $\mathrm{p}<0.05$ were then retained in the final model using a backwards elimination process. All of the statistical analyses were performed using Stata, release 11.0 (Stata Corp., TX, USA).

\section{Results \& discussion}

A total of 43 individuals with metastatic breast cancer had recordable CTC measurements with a median CTC count of 19 (range $0-4200$ cells/7.5 $\mathrm{ml}$ ), although the mean CTC count measured 311 cells $/ 7.5 \mathrm{ml}$. The majority had received multiple lines of previous chemotherapy and hormonal treatment $(>80 \%$ had estrogen receptor positive tumors), and had multiple metastatic sites (Table 1). Patients with very high CTC levels had an increased risk of immediate death. The median follow-up was 84 days and there were 29 events (deaths) during the study period.

The findings of the survival analysis suggested that patients with CTCs $>100$ ( $40 \%$ of the cohort) were at an increased risk of death compared with those with levels $\leq 100$ ( $60 \%$ of the cohort). Overall, the median overall survival in the former group measured 17.2 days (interquartile range 6.2-96.5 days) compared with 181.4 days (interquartile range 38.4 - not reached) in the latter (p $=0.009$, Log Rank test) (Figure 1). The findings of the exploratory Cox regression analysis revealed a hazard ratio of 3.1 for patients with CTCs $>100$ $(p=0.008,95 \%$ CI: 1.4-7.3). The only other prognostic variable that was retained in the model was number of lines of chemotherapy. There was a positive association between this variable and risk of death. The risk of death increased by $19 \%$ for each additional line of chemotherapy that the patient received ( $p=0.05$, HR: $1.19,95 \%$ CI: $1.0-1.4)$. None of the other variables were retained in the multivariable analysis.

Of the 50 tests performed, 7 'failed' to generate CTC measurements because collection of the sample proved problematic. Initial problems that resolved with time included: couriers failing to find the sample left at a specific location in a large teaching hospital; the sample being taken on a Thursday or Friday and arriving at the CTC testing center 120 miles away too late for weekday measurement; and an aborted CTC run on the machine. Such considerations are relevant 
Table 1. Patient characteristics for cohort 43 heavily pretreated individuals with metastatic breast cancer with 'no further therapeutic options' and a low performance status. Lines of previous therapy excludes adjuvant treatment. Individuals in whom circulating tumor cell measurement failed are not included.

\begin{tabular}{|c|c|}
\hline Age, median (range) & $56(34-88)$ years \\
\hline \multicolumn{2}{|l|}{ Chemotherapy $^{+}$} \\
\hline 1 line & 13 \\
\hline 2 lines & 6 \\
\hline 3 lines & 5 \\
\hline 4 lines & 6 \\
\hline 5 lines & 3 \\
\hline 6 lines & 4 \\
\hline 7 lines & 3 \\
\hline \multicolumn{2}{|l|}{ Hormonal treatment ${ }^{\neq}$} \\
\hline 1 line & 17 \\
\hline 2 lines & 6 \\
\hline 3 lines & 6 \\
\hline 4 lines & 4 \\
\hline 5 lines & 2 \\
\hline \multicolumn{2}{|c|}{ Number of metastatic sites ${ }^{\S}$} \\
\hline 1 site & 8 \\
\hline 2 sites & 13 \\
\hline 3 sites & 19 \\
\hline 4 sites & 3 \\
\hline \multicolumn{2}{|l|}{ Performance status } \\
\hline $0-1$ & 5 \\
\hline $2-3$ & 29 \\
\hline 4 & 9 \\
\hline \multicolumn{2}{|c|}{$\begin{array}{l}\text { 'Includes antibody therapy (trastuzumab, bevacizumab), small molecules (lapatinib) and bisphosphonates though administering } \\
\text { docetaxel + trastuzumab + zoledronic acid together, for example, is considered a single line of therapy. } \\
\text { †As per the above, hormonal therapy was often administered at the same time as chemotherapy and once again, this is considerec } \\
\text { a single line of therapy. } \\
\text { 'T }\end{array}$} \\
\hline
\end{tabular}

considering the cost of CTC measurements and the fixed cost of a machine, usually entailing that the facility which measures their levels will be at a different location to the one where the blood is collected from the patient.

It is not surprising that the risk of death increases with further lines of chemotherapy. Despite the low numbers of patients, the marked difference in survival between those with CTC counts above and below 100 indicates the utility of this test in this setting. The sample size is however too small to perform meaningful sensitivity/specificity analyses and future evaluations on larger sample sizes are required to not only assess predictive value, but also the CTC $>100$ cut off (another cut off may be better, i.e., more true positives). Interestingly, published data on file from Janssen Diagnostics describing a comprehensive analysis of correlations between disease progression and the lowest number of CTCs within a preset range across multiple test subjects with metastatic breast, prostate and colorectal showed a HR for a 100 CTC threshold of 6 . This compares to a HR of 3.1 for the same CTC threshold in our study. Differences here could be explained by the multiple tumor types in the Janssen data compared with individuals with breast cancer in this study. Based on our information thus far, individuals with a CTC count $>100$ can be advised that death is imminent, and further trials or drugs will not provide benefit. Conversely, those with a CTC count $<100$ had a much better prognosis than expected and patients and their loved ones can be advised accordingly. Recent data indicates a prominent theme of anxiety regarding the timing of diagnosing dying 'correctly', with an emphasis on how the dying patient and their families would cope if this were wrong [21]. Measurement of CTCs should improve the quality of end-of-life care by providing quantitative 


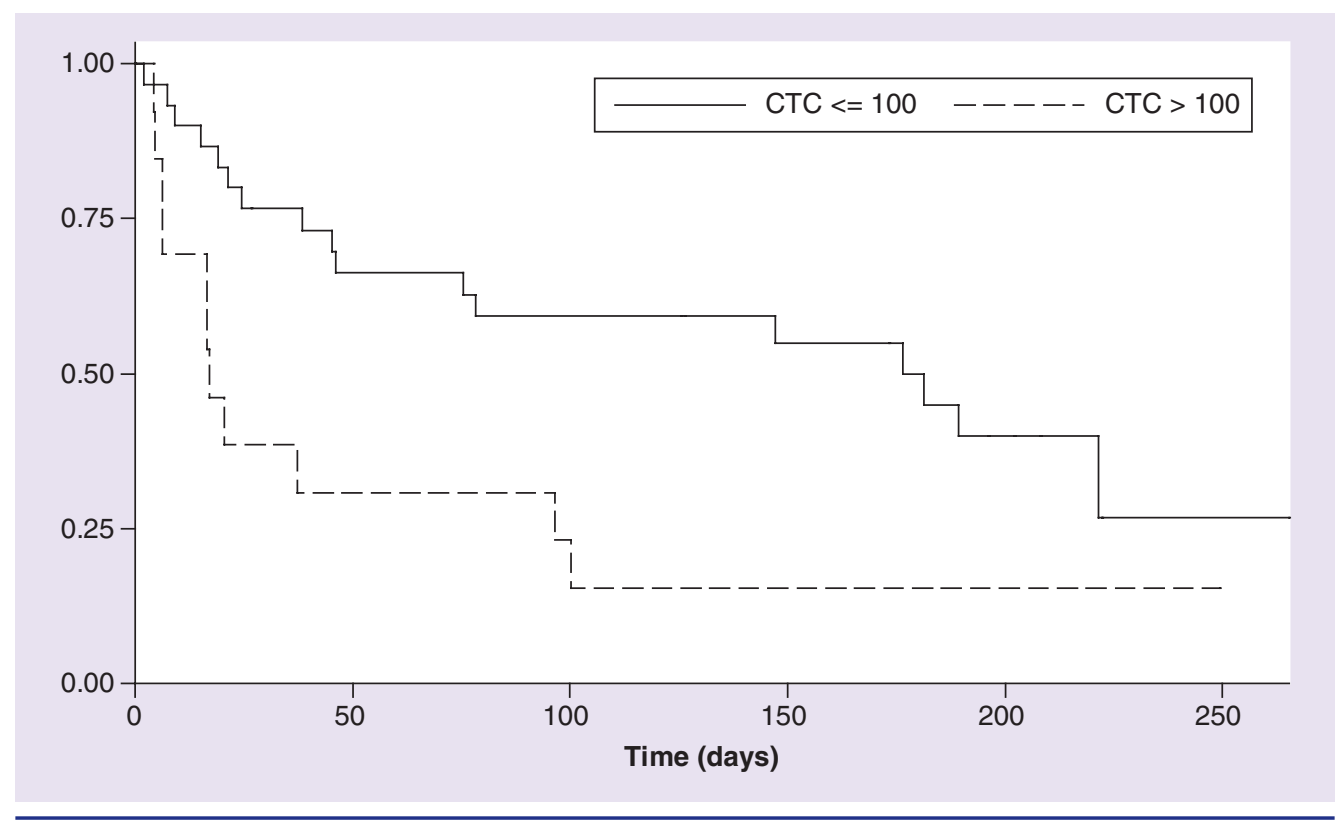

Figure 1. Kaplan-Meier survival curves demonstrating overall survival in individuals with $>100$ circulating tumor cells $(40 \%)$ versus those with $\leq 100$ circulating tumor cells $(60 \%)$. CTC: Circulating tumor cell.

prognostic information with immediate clinical utility, though we do not know if this is better than physician's 'best estimate' of life expectancy. Amongst the complexities of patient and family requirements at the end-of-life, such information is very likely to provide the foundations of evidence-based care that can be used as part of integrated pathways in clinical practice.

\section{Future perspective}

Many patients with metastatic cancer request information regarding their survival duration, especially when their deaths are approaching $[1,2]$, however we have few methods by which to accurately provide such information. There are data surrounding median survival for many tumor types and these can act as rough guides to prognosis but are generally based on histological grade and staging, are often suboptimal and do not provide an accurate or patient specific way of providing this information sufficiently. We therefore require a more precise prognostic tool to improve our ability to provide patients with a more accurate and realistic estimate of their life expectancy. Tumor burden may be an independent prognostic factor in some advanced malignancies, although not necessarily in breast cancer $[22,23]$, but there are a lack of data supporting a clear correlation between tumor burden and CTC levels. Interestingly a recent biomarker profiling study in a random group of patients assessed the prognostic power of a set of 106 candidate biomarkers for all-cause mortality and found that four biomarkers (alpha-1-acid glycoprotein, albumin, very-low-density lipoprotein particle size and citrate) were predictive of cardiovascular mortality, as well as death from cancer and other nonvascular diseases [24]. This study was not specific to cancer patients but provides further evidence for the use of biomarkers in this setting. CTCs have shown great promise as prognostic tools in early malignancy, and also have diagnostic potential and have become a standard test in many oncology clinics. However, until now their role as biomarkers for time to death has not been properly evaluated. Further larger studies are required but we believe that in the future, CTC measurements will also be utilized to provide patients with advanced malignancy with a more accurate estimate of their life expectancy.

Financial \& competing interests disclosure

The authors are grateful to Veridex for an unrestricted educational grant to provide 50 free circulating tumor cell tests. This work was supported by the Imperial ECMC and BRC.

The authors have no other relevant affiliations or financial involvement with any organization or entity with a financial interest in or financial conflict with the subject matter or materials discussed in the manuscript apart from those disclosed.

No writing assistance was utilized in the production of this manuscript. 


\section{EXECUTIVE SUMMARY}

\section{Introduction}

- The majority of individuals with metastatic cancer request information regarding their survival duration, especially when their deaths are approaching.

- There are a paucity of reliable tools for predicting survival duration although circulating tumor cell (CTC) measurement may prove to be a useful tool.

\section{Methodology}

- Between May 2011 and February 2012, consecutive individuals with metastatic breast cancer were considered eligible for CTC measurement provided two senior physicians considered they had less than 6 months to live. Patients included had an ECOG performance status of 2,3 or 4 and had exhausted conventional lines of therapy. Appropriate locoregional ethics committee approval was obtained.

- Statistical analyses were used to compare CTC levels with survival outcome measures.

\section{Results}

- Those with a CTC count of $\leq 100$ had a median of 182 days to live, compared with those with a CTC count of $>100$ who had a median of 17 days until death ( $p=0.009$, Log Rank, HR: 3.1, 95\% Cl: 1.4-7.3).

\section{Conclusion}

- A CTC count of $>100$ is associated with imminent death. Provided external validity is demonstrated, such information would be useful for patients and their families who often request specific prognostic clarity and could improve the quality of end-of-life care.

\section{References}

Papers of special note have been highlighted as:

- of interest; $\bullet \bullet$ of considerable interest

1 Hagerty RG, Butow PN, Ellis PA et al. Cancer patient preferences for communication of prognosis in the metastatic setting. J. Clin. Oncol. 22(9), 1721-1730 (2004).

- Relevant to the feelings of patients in terms of communication of prognosis.

2 Kiely BE, Tattersall MH, Stockler MR. Certain death in uncertain time: informing hope by quantifying a best case scenario. J. Clin. Oncol. 28(16), 2802-2804 (2010).

3 Hagerty RG, Butow PN, Ellis PM et al. Communicating with realism and hope: incurable cancer patients' views on the disclosure of prognosis. J. Clin. Oncol. 23(6), 1278-1288 (2005).

4 Gould SJ. The median is not the message. Discover 6, 40-42 (1985).

5 Davey HM, Butow PN, Armstrong BK. Cancer patients' preferences for written prognostic information provided outside the clinical context. Br. J. Cancer 89(8), 1450-1456 (2003).

6 Lobb EA, Butow PN, Kenny DT, Tattersall $\mathrm{MH}$. Communicating prognosis in early breast cancer: do women understand the language used? Med. J. Aust. 171(6), 290-294 (1999).
7 Francis PA. Surprised by hope. J. Clin. Oncol. 26(36), 6001-6002 (2008).

8 Stebbing J, Jiao LR. Circulating tumour cells as more than prognostic markers. Lancet Oncol. 10(12), 1138-1139 (2009).

9 Cristofanilli M, Budd GT, Ellis MJ et al. Circulating tumor cells, disease progression, and survival in metastatic breast cancer. N. Engl. J. Med. 351(8), 781-791 (2004).

10 Cristofanilli M, Hayes DF, Budd GT et al. Circulating tumor cells: a novel prognostic factor for newly diagnosed metastatic breast cancer. J. Clin. Oncol. 23(7), 1420-1430 (2005).

- One of the earliest studies showing a role for circulating tumor cells (CTCs) as prognostic biomarkers.

11 Budd GT, Cristofanilli M, Ellis MJ et al. Circulating tumor cells versus imaging predicting overall survival in metastatic breast cancer. Clin. Cancer Res. 12(21), 6403-6409 (2006).

12 Giordano A, Egleston BL, Hajage D et al. Establishment and validation of circulating tumor cell-based prognostic nomograms in first-line metastatic breast cancer patients. Clin. Cancer Res. 19(6), 1596-1602 (2013).

13 Krell J, Stebbing J. Circulating tumour cells as biomarkers in early breast cancer. Lancet Oncol. 13(7), 653-654 (2012).
14 Lucci A, Hall CS, Lodhi AK et al. Circulating tumour cells in non-metastatic breast cancer: a prospective study. Lancet Oncol. 13(7), 688-695 (2012).

15 Franken B, de Groot MR, Mastboom WJ et al. Circulating tumor cells, disease recurrence and survival in newly diagnosed breast cancer. Breast Cancer Res. 14(5), R133 (2012).

16 Bidard FC, Belin L, Delaloge S et al. Time-dependent prognostic impact of circulating tumor cells detection in non-metastatic breast cancer: 70 -month analysis of the REMAGUS02 study. Int. J. Breast Cancer 2013, 130470 (2013).

-. One of the largest studies demonstrating the potential clinical utility of CTCs.

17 Jiao LR, Apostolopoulos C, Jacob J et al. Unique localization of circulating tumor cells in patients with hepatic metastases. J. Clin. Oncol. 27(36), 6160-6165 (2009).

18 Payne RE, Yague E, Slade MJ et al. Measurements of EGFR expression on circulating tumor cells are reproducible over time in metastatic breast cancer patients. Pharmacogenomics 10(1), 51-57 (2009).

19 Pestrin M, Bessi S, Puglisi F et al. Final results of a multicenter Phase II clinical trial evaluating the activity of single-agent lapatinib in patients with HER2-negative metastatic breast cancer and HER2-positive circulating tumor cells. A proof-of-concept 
PRELIMINARY COMMUNICATION Stebbing, Harding, Urch et al.

study. Breast Cancer Res. Treat.134(1), 283-289 (2012).

20 Graham ML, Fleming N, Pinto A, Dropkin E. Circulating tumor cells (CTCs) and association with survival in molecular subtypes (MST) of metastatic breast cancer (MBC). J. Clin. Oncol. 28(Suppl. 15), 1094 (2010).

21 Dee JF, Endacott R. Doing the right thing at the right time. J. Nurs. Manag. 19(2), 186-192 (2011).
22 Andersson Y, Frisell J, Sylvan M de Boniface J Bergkvist L. Breast cancer survival in relation to the metastatic tumor burden in axillary lymph nodes. Journal Clinical Oncology. 28(17), 2868-2873 (2010).

23 Lacovelli R, Lanoy E, Albiges L, Escudier B. Tumour burden is an independent prognostic factor in metastatic renal cell carcinoma.. BJU Int. 110(11), 1743-1753 (2012).
24 Fischer K, Kettunen J, Würtz P et al. Biomarker profiling by nuclear magnetic resonance spectroscopy for the prediction of all-cause mortality: an observational study of 17,345 persons. PLoS Med. 11(2), e100160100166 (2014). 